\title{
Symmetrical orientation of spiral-interconnects for high mechanical stability of stretchable electronics
}

\author{
Nadeem Qaiser ${ }^{1}$, Asrar Nabil Damdam ${ }^{1}$, Sherjeel Munsif Khan', Muhammad Mustafa Hussain ${ }^{1,2^{*}}$ \\ ${ }^{1} \mathrm{mmh}$ labs, Electrical Engineering, Computer Electrical and Mathematical Science and Engineering Division, King Abdullah \\ University of Science and Technology (KAUST) Thuwal, 23955, Saudi Arabia. \\ ${ }^{2}$ EECS, University of California, Berkeley, CA 94720, USA. \\ *mmhussain@berkeley.edu / muhammad.hussain@kaust.edu.sa
}

\begin{abstract}
Recently, interconnect based stretchable electronic devices have attained growing interest due to its application for various state-of-the-art technologies. Here, we report an engineered design of spiral interconnects for a series of stretchable networks referred to as the symmetrical series; wherein spirals connect to the island in the symmetry manner. A systematic analysis of Si-based spiral interconnects by numerical modeling, and experiments show that our design provides higher stretchability of $165 \%$ in comparison to the conventionally used nonsymmetrical design. The reason for high mechanical reliability is attributed to the favorable unwrapping profile of spiral interconnect due to the nature of forces acting on it during the stretching process. In contrast, for the nonsymmetrical series, the nature of tensile forces produces the rotation, and resultant tilting of spiral arm results in low stretchability of $150 \%$. As a result, nonsymmetrical interconnect fails at earlier stages of stretching. Our study demonstrates the significance of the orientation of spiral interconnects linked to the island to attain the high performance of stretchable electronic devices.
\end{abstract}

Keywords-stretchable electronics; numerical analysis; microfabrication; symmetry; spiral interconnect

\section{INTRODUCTION}

Currently, using thin silicon (Si) and metallic-based mechanically compliant, stretchable, and reconfigurable electronic devices provides the way to realize state-of-the-art applications ranging from wearable, flexible, and soft sensory robotics, e-skin, optoelectronics, biomedical devices, and other reconfigurable display [1]-[7]. Another perspective consists of integrating the required components with the human tissues for the sake of collecting information on health vitals and taking the associated clinical procedures [8]-[10]. Achieving high performance of these stretchable devices with low cost would only be possible by apprehending the detailed mechanical response of these devices during their functioning, i.e., mechanical deformation and evolved stress during the stretching process. Based on numerical and experimental studies, researchers have suggested various approaches to get the desired functionality without failure or crack during stretching [11], [12]. One of these strategies includes using geometrically stretchable designs, including the fractals, serpentine, and spiral [13]-[17]. These stretchable configurations utilize the model where an island holds all of the functional devices and interconnect connected to the islands act as a buffer to retain the stretchability. For instance, studies have shown a remarkable mechanical performance by employing islandinterconnect configurations [18], [19]. Among others, the geometry of the spiral as an interconnect provides high stretchability by unwrapping its arm from the inner circle. Although studies have shown the mechanical response of spiral-based interconnects, the orientation of spiral itself or the way spiral-interconnect attaches to the island was chosen arbitrarily. Despite the significance, previous studies lack the understanding of the spiral orientation or way it links to the respective islands. Here, we report the importance of the order of linkage of a spiral-interconnect to the island. We chose a single basic unit cell as a representative of complex square or orthogonal array and proposed a symmetrical arrangement around the centerline. A systematic FEM modeling and experimental results show the benefits of the proposed design as compared to conventionally used nonsymmetrical design. For instance, our proposed symmetrical-orientation of the spiral interconnect provides high stretchability as compared to the conventionally used counterparts. Likewise, the stress at the spiral's arm for conventionally used nonsymmetrical series was found to be $163 \%$ higher due to rotational forces or tilting effect on the spiral-interconnect that enhances the mechanical reliability. Our design could be extended to the complex square-array fashion, i.e., linking of multiple islands and spirals to form a square-shape, [19] which can be used in an advanced stretchable electronic system.

\section{MATERIALS AND METHODS}

\section{A. Numerical modeling}

We conducted the finite element method (FEM) analysis that did mimic the mechanical response, i.e., mechanical deformation, stress, and strain of our proposed symmetrical island-interconnect stretchable series and compared it with the conventionally used nonsymmetrical series. We used an FEA tool, ABAQUS ${ }^{\mathrm{TM}}$, that is commercially available. Both of the geometries were designed in SOLIDWORKSTM with an extruded thickness of $20 \mu \mathrm{m}$ and then imported to the FEM environment for further study. We prescribed in-plane displacement $D_{p}$, at right $\left(\mathrm{I}_{1}\right)$, and left-sided island $\left(\mathrm{I}_{3}\right)$ of series (shown in figure 1). Each design was stretched up to the point where the maximum induced principal strain reached the intrinsic fracture strain of $1 \%$ for Si. To account for the large deformations, i.e., geometrical nonlinearities, "NLgeom on" was employed in ABAQUSTM. A refined element was chosen to ensure the solution's convergence. The elastic analysis was used for $\mathrm{Si}$ with material properties taken from previous work [20]. 


\section{B. Fabrication of Si-based spiral interconnects}

To validate the benefits of our design, we fabricated the Si-based stretchable series and tested for uniaxial stretching. We deposited the layer of oxide $\left(\mathrm{SiO}_{2}\right)$ of a thickness of 10 $\mu \mathrm{m}$ and amorphous silicon $(a-\mathrm{Si})$ of a thickness of $20 \mu \mathrm{m}$ on Si wafer by using PECVD (Oxford Instruments PlasmaLab Deposition). A hard mask with a thickness of $200 \mathrm{~nm}$ of Aluminum (Al) was sputtered (Equipment Support Company). Patterning was achieved by Lithography (EVG 620S). Al was etched by using metal reactive ion etching (RIE). Next, deep RIE (Oxford Instruments PlasmaLab 100) was used to attain a thickness of $20 \mu \mathrm{m}$ of $a$-Si. Before the final release of the design, a wet-etchant (for five minutes) was employed to remove the hard mask of Al. Finally, $48 \%$ HF, i.e., Hydrofluoric acid (Sigma-Aldrich) was used to perform the wet etching for 1 hour at room temperature that released the final structure. The fabricated samples were dried and stretched uniaxially until they break by using the probe-testing machine.

\section{RESULTS AND DISCUSSION}
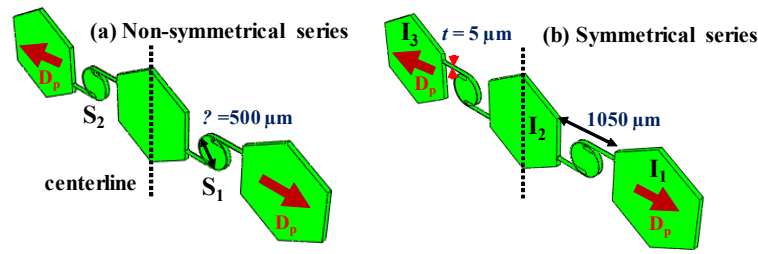

Fig. 1. Schematics of (a) conventionally used and (b) our proposed symmetrical series of stretchable spiral interconnects with associated parameters.

We chose island-interconnect configuration, wherein the island holds all of the functionalities, and the spiral interconnect provides a significant amount of stretchability by accommodating the large deformations during stretching. For a fully stretchable system, especially when islands and spirals are connected in a square-array fashion [19], taking a simple unit cell (Fig. 1) in-series, i.e., spiral interconnects $S_{1}$ and $S_{2}$ connected in series with $I_{1}, I_{2}$, and $I_{3}$ will suffice to mimic the in-plane stretching of full array. Fig. 1a illustrates the schematics of conventionally used nonsymmetrical series. Fig. $1 \mathrm{~b}$ shows the proposed symmetrical series, i.e., spirals are oriented or connected to the islands in symmetry or represent a mirror topology around a centerline, which will provide better mechanical stability due to its particular engineered design. The parameters of the stretchable series are also shown in Fig. 1. The thickness (t) and diameter of the inner circle of the spiral $(\phi)$, and arm end-to-end distance/gap between two islands are considered as $5 \mu \mathrm{m}$, $500 \mu \mathrm{m}$, and $1050 \mu \mathrm{m}$, respectively.

To demonstrate the advantage of our symmetrical series in terms of mechanical stability, i.e., deformations and stress/strains, we first employ the numerical analysis. As the spirals are connected in series, each spiral experiences an identical magnitude of deformation, stress, and strain. Fig. 2 shows the comparison of the symmetrical series with the nonsymmetrical counterparts. Fig. $2 \mathrm{a}$ and $2 \mathrm{~b}$ represent the initial undeformed geometries, i.e. when no stretching is experienced. Upon uniaxial stretching along the $\mathrm{x}$-axis, $\mathrm{I}_{1}$ and $\mathrm{I}_{3}$ move axially. Interestingly, due to symmetrical orientation or collinear forces exerted by $S_{1}$ and $S_{2}$, the $I_{2}$ moves vertically that relaxes the deformation caused by tensile stretching, as shown in Fig. 2c. In other words, the end of the arm connected to $\mathrm{I}_{2}$ does permit further stretching/unwrapping of spirals. In contrast, conventionally used nonsymmetrical series experiences the anticlockwise rotation or torque due to the nature of the line of forces. The resultant rotation exerts the additionally tilting at the end of the spiral's arm connected to the $I_{2}$ that, in turn, elevates the stress of spiral to the fracture limit at earlier stages of stretching. Fig. 2e and $2 \mathrm{f}$ reveal stress when spiral interconnects reach the fracture conditions [11], [21]. Insets show the $3 \mathrm{D}$ stress contours at the principal strain of $1 \%$.
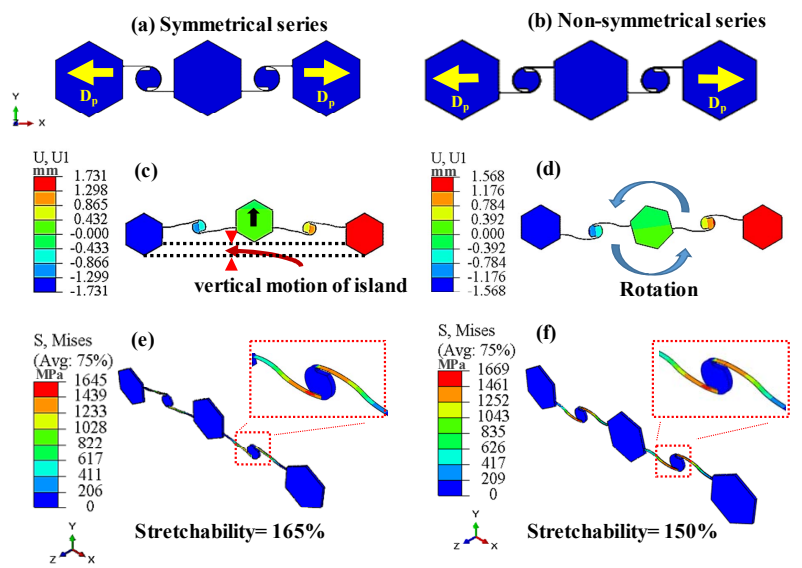

Fig. 2. Mechanics of symmetrical and nonsymmetrical series at the onset of fracture conditions by FEM. (a, b) Initial undeformed states, (c) symmetry results in the upward motion of the middle island due to the nature of evolved forces, and (d) rotation of conventionally used series causes tilting. (e, f) 3D stress contours of von Mises stress for both cases. Results show the higher stretchability (of 165\%) for the proposed symmetrical series as compared to nonsymmetrical counterparts (of 150\%).
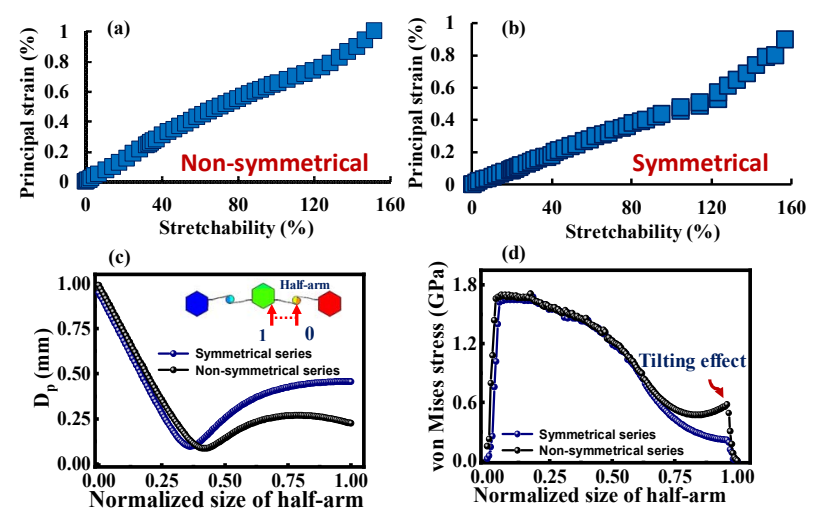

Fig. 3. (a, b) Evolution of principal strain as a function of stretching for both cases. Along the half-arm, (c) displacement distribution. The definition of half-arm is shown in the inset. The elevated $D_{p}$ for symmetrical series is caused by the upward movement of $\mathrm{I}_{2}$. (d) The stress distribution of the arm. For conventionally used series, spiral's arm experiences stress of $0.58 \mathrm{GPa}$ as compared to $0.22 \mathrm{GPa}$ for symmetrical series, which is $163 \%$ lower than nonsymmetrical series making our design mechanically more stable.

Results show that the stretchability of the symmetrical orientation is higher $(\sim 165 \%)$ as compared to the conventionally used nonsymmetrical design $(\sim 150 \%)$, showing improved mechanical stability of our symmetricalorientated spiral interconnects when used for electronic devices. Fig. $3 \mathrm{a}$ and $3 \mathrm{~b}$ demonstrate the evolution of principal strain as a function of stretchability, showing the maximum achievable stretchability for each of the design at 
the onset of $1 \%$ strain, i.e., intrinsic fracture limit of Si. Next, as the mechanical behavior of the spiral along the boundary of the arm is crucial to fully comprehend the mechanics of the stretchable system during its functioning. Therefore, it will be worthy of mapping the $\mathrm{D}_{\mathrm{p}}$ and von Mises stress along the arm. As the stress contours shown in Fig. 2 validate that stress in the arm is identical around the inner circle, we define half-arm from 0 to 1 , as arm's end connected to inner circle and $\mathrm{I}_{2}$, respectively. The half-arm is shown in the inset of Fig. 3c. Fig. 3c shows the $D_{p}$ along the arm for symmetrical and nonsymmetrical series. As discussed above, our design results in higher stretching or extended opening of the spiral. Fig. $3 d$ shows the stress along the arm of the spiral. Results demonstrate at the position of 1 (of the normalized size of half-arm), that conventionally used design evolves the $163 \%$ higher stress due to the tilting effect at the arm's end connected to $I_{2}$, which is expected to reduce the

(a) Fabrication flow

(b)

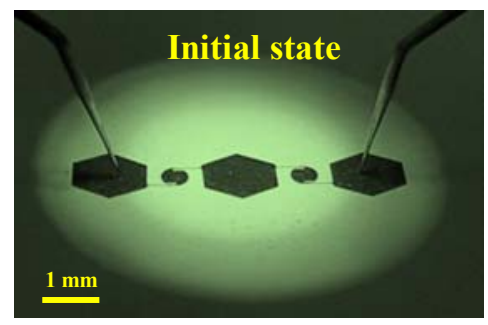

(d)

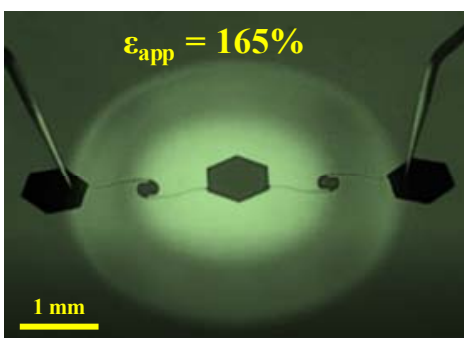

(c)

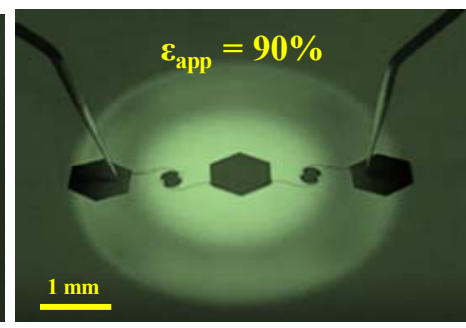

(e)

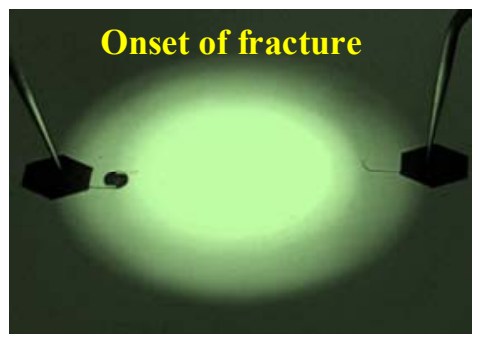

Fig. 4. Fabrication process and experimental tensile testing of the symmetrical series. (a) Fabrication flow showing the steps taken to manufacture the micron-sized samples. The parameters in the numerical modeling and fabricated samples were taken as comparable. (b-d) The stretching or unwarping profile of spiral interconnects from initial deformed state to stretching of $90 \%, 165 \%$, and at the failure.

\section{CONCLUSIONS}

In summary, we report an engineered design of the spiral interconnects series containing the symmetrical orientation of spiral around the islands that provides the higher stretchability and mechanical stability for high performance stretchable electronic devices. In contrast, the conventionally used nonsymmetrical design has a lower stretchability of $150 \%$ as compared to $165 \%$ stretchability of our symmetrical design since the spiral of nonsymmetrical series exceeds the fracture limit at earlier stages of stretching due to the tilting effect. Additionally, the spiral's arm region for conventionally used series near to the middle-island has a von Mises stress of $0.58 \mathrm{GPa}$ as compared to $0.22 \mathrm{GPa}$ for symmetrical series, which is $163 \%$ lower than nonsymmetrical series. We reveal the underlying mechanism of the higher stretchability of symmetrical series by using numerical modeling, and then results were validated by testing the fabricated samples of Si. Our results suggest that the orientation of the interconnect when connected to the islands could play a crucial role and demands further detailed testing and study for full complex stretchable arrays.

\section{REFERENCES}

[1] N. Qaiser, S. M. Khan, K. Chow, M. D. Cordero, I. Wicaksono, and M. M. Hussain, "3D Printed Robotic Assembly Enabled Reconfigurable Display with Higher Resolution," Adv. Mater. Technol., vol. 3, no. 12, Dec. 2018.

[2] J. A. Rogers, T. Someya, and Y. Huang, "Materials and Mechanics for Stretchable Electronics," Science (80-. )., vol. 327, no. 5973, 2010.

[3] S. F. Shaikh et al., "Noninvasive Featherlight Wearable Compliant 'Marine Skin': Standalone Multisensory System for Deep-Sea Environmental Monitoring," Small, vol. 15, no. 10, p. 1804385 , Mar. 2019.

[4] D. H. Kim, J. Xiao, J. Song, Y. Huang, and J. A. Rogers, "Stretchable, curvilinear electronics based on inorganic materials," Advanced Materials, vol. 22, no. 19. pp. 2108-2124, 18-May-2010.

[5] S. $\mathrm{Xu}$ et al., "Soft Microfluidic Assemblies of 
Sensors, Circuits, and Radios for the Skin," Science (80-. )., vol. 344, no. 6179, 2014.

[6] K. Zhang et al., "Origami silicon optoelectronics for hemispherical electronic eye systems," Nat. Commun., vol. 8, no. 1, p. 1782, Dec. 2017.

[7] G. A. Torres Sevilla, N. Qaiser, M. D. Cordero, S. F. Shaikh, and M. M. Hussain, "Fully spherical stretchable silicon photodiodes array for simultaneous 360 imaging," Appl. Phys. Lett., vol. 113, no. 13, p. 134101, Sep. 2018.

[8] W. Gao et al., "Fully integrated wearable sensor arrays for multiplexed in situ perspiration analysis," Nature, vol. 529, no. 7587, pp. 509-514, Jan. 2016.

[9] S. Emaminejad et al., "Autonomous sweat extraction and analysis applied to cystic fibrosis and glucose monitoring using a fully integrated wearable platform," Proc. Natl. Acad. Sci., vol. 114, no. 18, pp. 4625-4630, May 2017.

[10] C. Choi et al., "Human eye-inspired soft optoelectronic device using high-density MoS2graphene curved image sensor array," Nat. Commun., vol. 8, no. 1, p. 1664, Dec. 2017.

[11] J. A. Fan et al., "Fractal design concepts for stretchable electronics," Nat. Commun., vol. 5, p. 3266, Feb. 2014.

[12] Y. Su et al., "Elasticity of Fractal Inspired Interconnects," Small, vol. 11, no. 3, pp. 367-373, Jan. 2015.

[13] A. M. Hussain, E. B. Lizardo, G. A. Torres Sevilla, J. M. Nassar, and M. M. Hussain, "Ultrastretchable and flexible copper interconnect-based smart patch for adaptive thermotherapy," Adv. Healthc. Mater., vol. 4, no. 5, pp. 665-673, Apr. 2015.

[14] Z. Fan et al., "A finite deformation model of planar serpentine interconnects for stretchable electronics,"
Int. J. Solids Struct., vol. 91, pp. 46-54, Aug. 2016.

[15] Y. Su, Z. Liu, S. Kim, J. Wu, Y. Huang, and J. A. Rogers, "Mechanics of stretchable electronics with high fill factors," Int. J. Solids Struct., vol. 49, no. 23-24, pp. 3416-3421, Nov. 2012.

[16] C. Lv, H. Yu, and H. Jiang, "Archimedean spiral design for extremely stretchable interconnects," 2014.

[17] M. Gonzalez, F. Axisa, M. Vanden Bulcke, D. Brosteaux, B. Vandevelde, and J. Vanfleteren, "Design of metal interconnects for stretchable electronic circuits," Microelectron. Reliab., vol. 48, no. 6, pp. 825-832, Jun. 2008.

[18] N. Qaiser, S. M. Khan, and M. M. Hussain, "Inplane and out-of-plane structural response of spiral interconnects for highly stretchable electronics," $J$. Appl. Phys., vol. 124, no. 3, p. 034905, Jul. 2018.

[19] N. Qaiser, A. N. Damdam, S. M. Khan, S. F. Shaikh, and M. M. Hussain, "Design, mechanics, and operation of spiral-interconnect based networked sensor for stretchable electronics," Appl. Phys. Lett., vol. 115, no. 18, Oct. 2019.

[20] W. Cheng et al., "Fast-Response and LowHysteresis Flexible Pressure Sensor Based on Silicon Nanowires," IEEE Electron Device Lett., vol. 39, no. 7, pp. 1069-1072, Jul. 2018.

[21] N. Qaiser, S. M. Khan, M. Nour, M. U. Rehman, J. P. Rojas, and M. M. Hussain, "Mechanical response of spiral interconnect arrays for highly stretchable electronics," Appl. Phys. Lett., vol. 111, no. 21, p. 214102, Nov. 2017. 